\title{
Head motion during overt language production in functional magnetic resonance imaging
}

\author{
Stefan Heim ${ }^{\mathrm{a}, \mathrm{b}}$, Katrin Amunts ${ }^{\mathrm{a}, \mathrm{c}}$, Hartmut Mohlberg ${ }^{\mathrm{a}, \mathrm{b}}$, Marcus Wilms ${ }^{\mathrm{a}}$ and Angela D. Friederici ${ }^{\mathrm{d}}$ \\ ${ }^{a}$ Research Centre Jülich, Institute of Medicine, ${ }^{b}$ Brain Imaging Center West (BICW), Jülich, ${ }^{c}$ Department of Psychiatry and Psychotherapy, \\ RWTH Aachen and ${ }^{d}$ Max Planck Institute for Human Cognitive and Brain Sciences, Leipzig, Germany
}

Correspondence and requests for reprints to Dr Stefan Heim, Research Centre Jülich, Institute of Medicine, AG Brain Mapping, 52425 Jülich, Germany Tel: + 49246161 4208; fax: + 49246161 2820; e-mail: s.heim@fz-juelich.de

\begin{abstract}
Sponsorship: Research was supported by the Max Planck Society (SH) and the Human Brain Project/Neuroinformatics research funded by the National Institute of Biomedical Imaging and Bioengineering, the National Institute of Neurological Disorders and Stroke, and the National Institute of Mental Health (K.A.).
\end{abstract}

Received 16 January 2006; accepted 8 February 2006

\begin{abstract}
Head motion parameters (translation, rotation) during an eventrelated functional magnetic resonance imaging experiment of overt picture naming were investigated for two fixation conditions. We compared normal fixation with cushions and belts with the additional use of a bite-bar. Neither mean nor maximum values differed between the two conditions for any of the parameters. By applying the head motion parameters to the cytoarchitectonically
\end{abstract}

defined volume of the left Brodmann area 44, we demonstrated volume overlap of $95 \%$ (normal fixation) to $100 \%$ (bite-bar) that did not differ significantly between fixation conditions. The data encourage further use of overt language production in functional magnetic resonance imaging with careful head fixation but no bite-bar. NeuroReport 17:579-582 (c) 2006 Lippincott Williams \& Wilkins.

Keywords: Broca's area, Brodmann area 44, functional magnetic resonance imaging, head movement, language, production, speech

\section{Introduction}

The investigation of brain activity during overt utterances has become increasingly popular in recent years (for a recent review of object naming studies, cf. Ref. [1]). Whereas most of the earlier studies employed positron emission tomography and blocked designs, functional magnetic resonance imaging (fMRI) was less frequently used, mainly because of the expected large head motions and the susceptibility artefacts caused by this motion. Whereas more recent studies have now demonstrated the general feasibility of employing overt language production in fMRI [2-8], information about effective head fixation methods [4], the absolute maximum head motion, and the actual dislocation of language-relevant brain regions such as Broca's area during speaking is still wanting. Such information is crucial for the planning and interpretation of future fMRI studies using overt speech paradigms, for example the Stroop task, reading aloud, or picture naming.

Therefore, we quantified the mean and the maximum head motion parameters in an event-related fMRI experiment of overt picture naming (data from Ref. [6]) employing two different head fixation conditions, standard fixation vs. standard fixation plus a bite-bar. Moreover, we demonstrate the amount of brain volume displacement in the languagerelevant Broca's region during speaking on the basis of the cytoarchitectonic probability map of Brodmann area (BA) 44. The results may be useful for the design of future studies employing overt speech production in fMRI.

\section{Methods}

The experimental procedure has been described in detail in Ref. [6]. Twelve study participants (six women; age 21-30 years) saw line drawings of real objects and overtly produced the German picture name or its definite determiner or said 'jaja' when a smiley was presented as a baseline condition. A total of 1070 functional echo planar imaging volumes $\left(18 \mathrm{~min} ; \mathrm{TR}=1 \mathrm{~s}, \mathrm{TE}=30 \mathrm{~ms}\right.$, flip angle $=90^{\circ}$, $\mathrm{FOV}=25.0 \mathrm{~cm}$ ) were acquired at $3 \mathrm{~T}$ (Medspec 30/100, Bruker, Ettlingen, Germany) from 12 sagittal slices (thickness $3 \mathrm{~mm}$, gap $1 \mathrm{~mm}$ ) in the left hemisphere. All participants received a standard head fixation with cushions placed in the standard birdcage head coil and belts mounted onto the same coil. Whereas one group received only this fixation (FIX), five participants additionally had to bite on a bar mounted on the head coil (BITE). Both groups were matched $(P>0.05)$ with respect to sex (FIX: four women, three men; BITE: two women, three men) and age (FIX: 24.6 years; BITE: 24.0 years). The experimental standards were approved by the local Ethics Committee of the University of Leipzig. Informed consent was obtained from all participants.

Parameters for translation (parallel to the $x, y$, and $z$-axis) and rotation around these axes (pitch, roll, and yaw; Fig. 1) obtained from the rp*.txt outputs of the SPM2 realignment function were analysed. For each participant and parameter, the mean and the maximum were calculated. These data entered a group analysis for the overall mean (mean), mean of maximum $[\operatorname{mean}(\max )]$, and overall maximum 


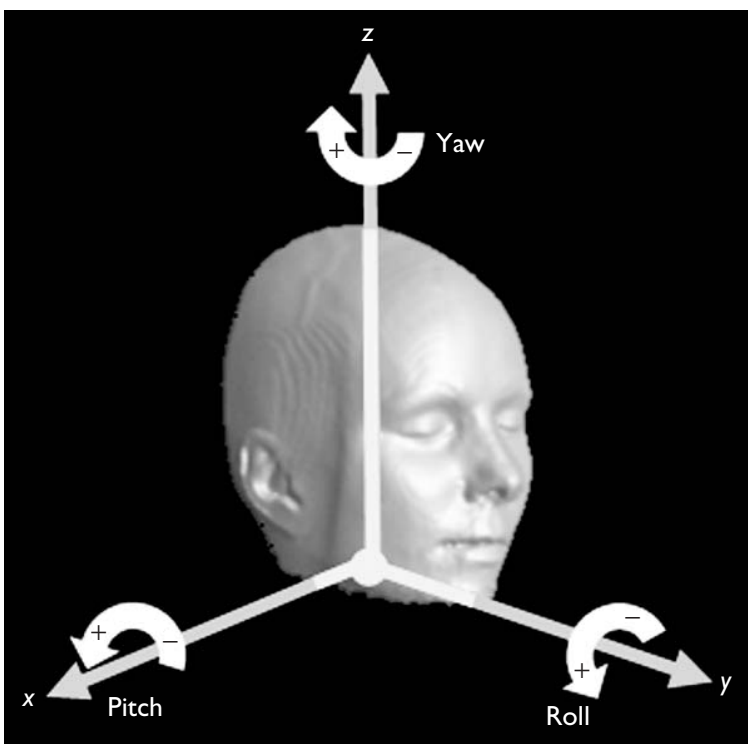

Fig. I Schematic display of the three translation dimensions $(x, y, z$; arrows point to the direction of positive coordinates) and the three rotation directions (pitch, around $x$-axis; roll, around $y$-axis, and yaw, around $z$-axis). $[\max (\max )]$. The group analysis was carried out for the total sample and separately for the two fixation conditions. The difference of the mean(mean) and mean(max) values of both groups were then tested (Mann-Whitney $U$-test).

Moreover, we tested the amount of head motion in terms of the displacement of a three-dimensional brain region during speaking. We selected BA 44 in Broca's speech region, which was significantly activated during the picture naming task [7] (Fig. 2). The motion parameters were applied to the cytoarchitectonically defined probability map of the left BA $44[9,10]$. For each participant, the location and volume of BA 44 before $\left(V_{0}\right)$ and after $\left(V_{1}\right)$ the fMRI scan were assessed. From these data, the percentage overlap $\Omega$ of both volumes was calculated [cf. Equation (1)].

$$
\text { Volume overlap } \Omega=\frac{V_{0}-V_{1}}{V_{0}} \times 100 \% .
$$

Finally, a group analysis of the mean overlap was conducted for the total sample and separately for the FIX and BITE groups.

\section{Results}

Figure 2 shows the fMRI results for the contrast 'Picture naming $>$ Null events' for the total sample and separately

Picture naming $>$ Null events
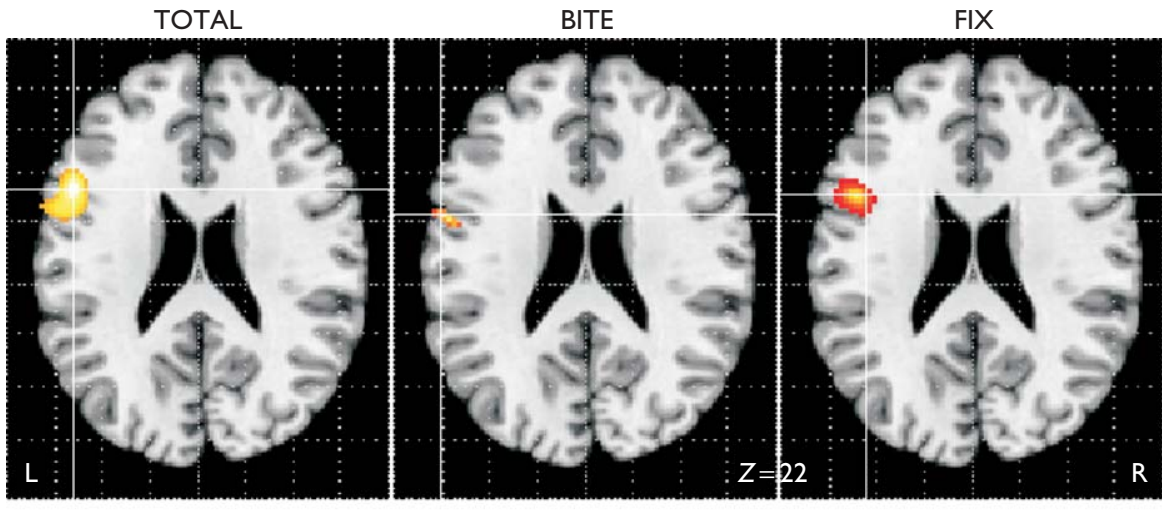

$$
0<T<5
$$

$0<T<9$

$0<T<10$

Fig. 2 SPM $\{T\}$ displays of the functional brain activation in the contrast 'Picture naming $>$ Null events' reveal activation in Broca's region for the total sample and the two head fixation conditions. For visualization purposes, the threshold was set to $P<0.01$ (uncorrected).

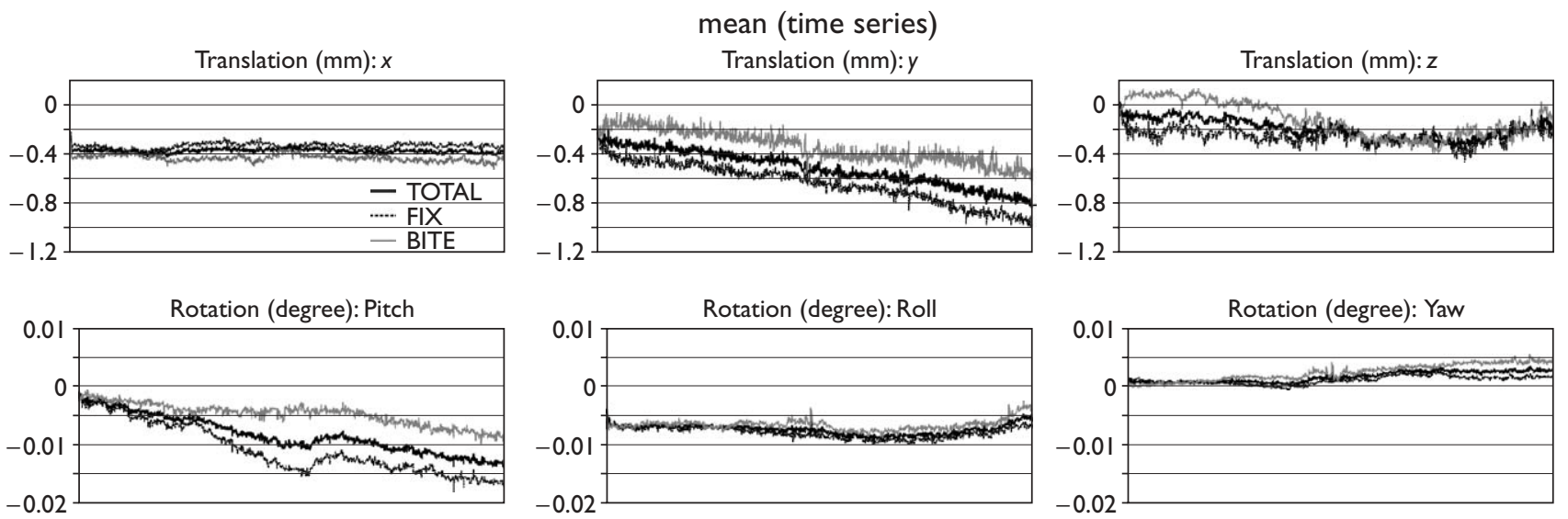

Fig. 3 Group means of the time series of the head motion parameters for the total sample (TOTAL, bold solid black line), the fixation-only group (FIX, black dashed line), and the fixation-plus-bite-bar group (BITE, solid grey line). 

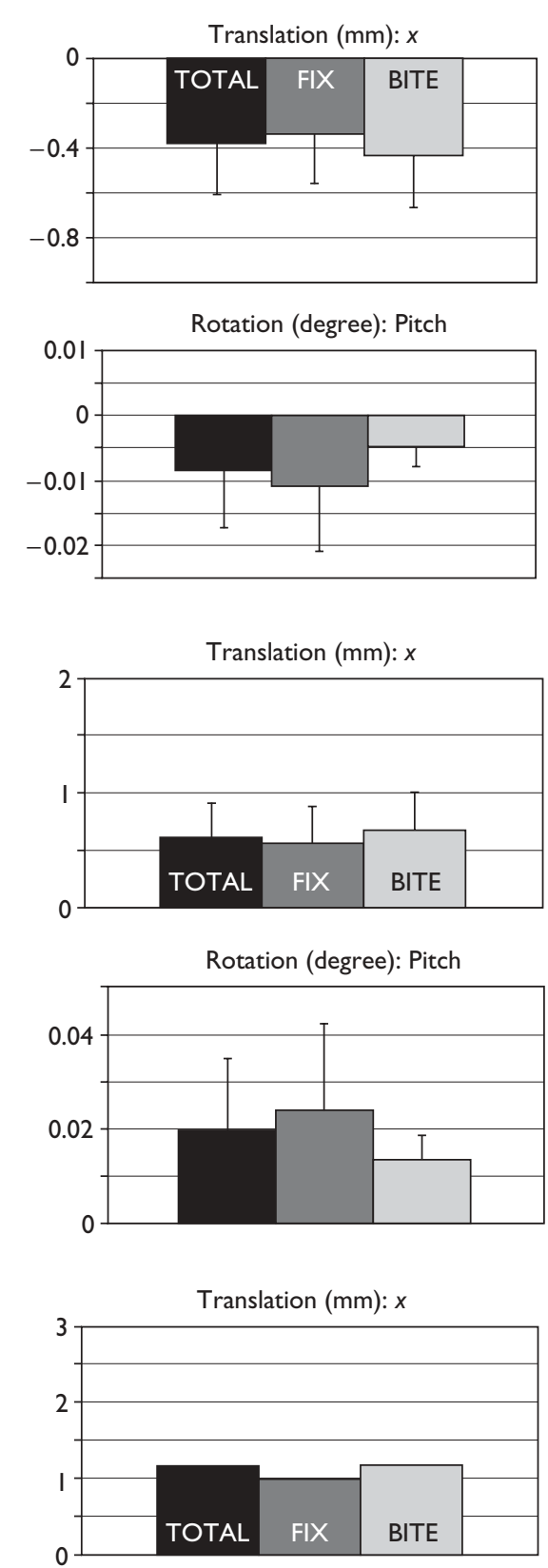

Rotation (degree): Pitch

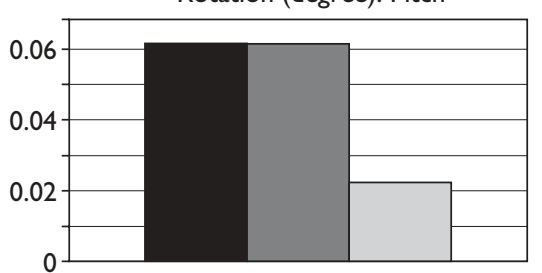

mean (mean)

Translation (mm): $y$
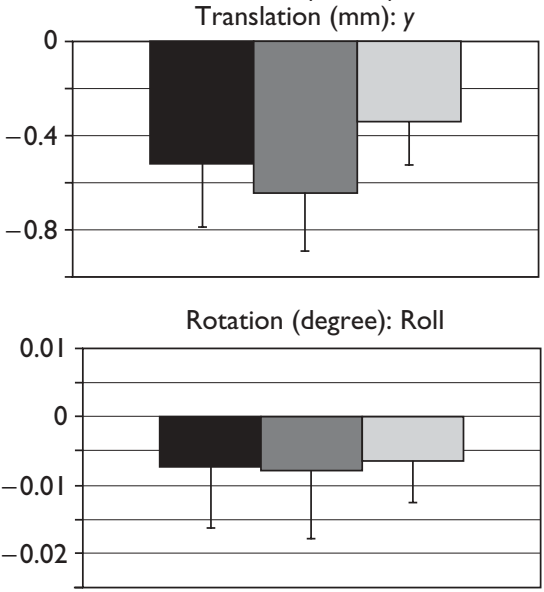

mean (max)

Translation (mm): $y$

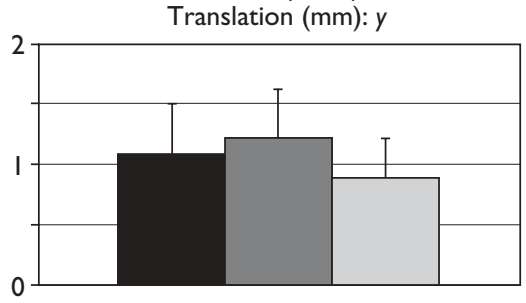

Rotation (degree): Roll

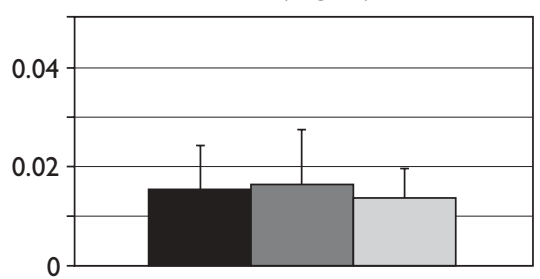

$\max (\max )$

Translation (mm): $y$

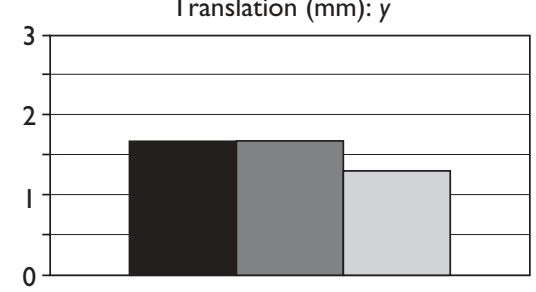

Rotation (degree): Roll

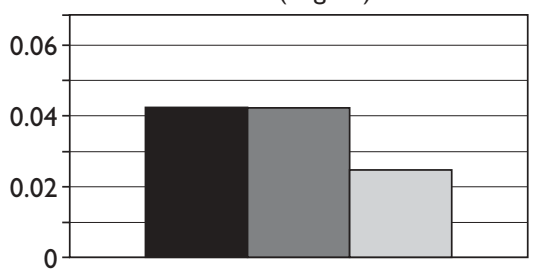

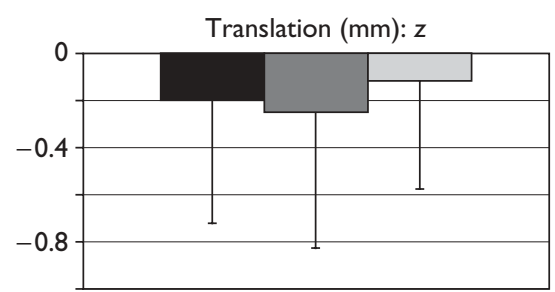

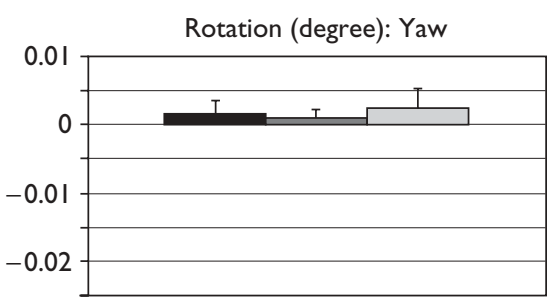

Translation (mm): z

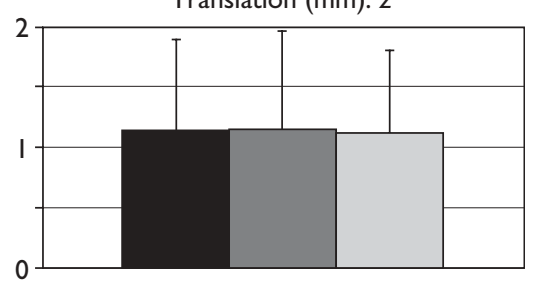

Rotation (degree): Yaw

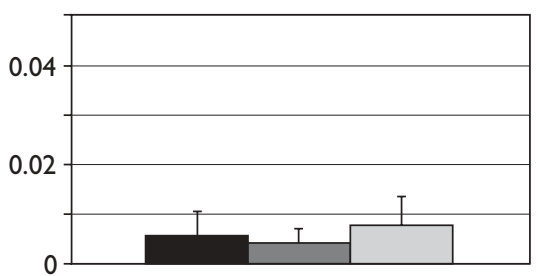

Translation (mm): z

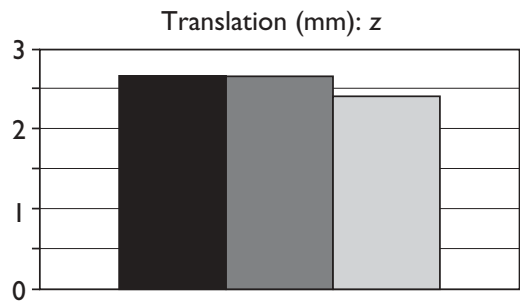

Rotation (degree): Yaw

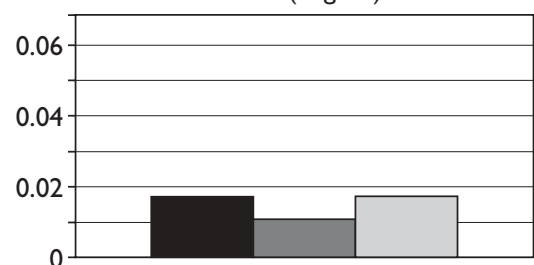

Fig. 4 Analysis of the translation $(x, y, z)$ and rotation (pitch, roll, yaw) parameters for the total sample (TOTAL, black graphs), the fixation-only group (FIX, dark grey), and the fixation-plus-bite-bar group (BITE, light grey). Upper panel: group means of participants' mean. Middle panel: group means of participants' maxima. Lower panel: absolute group maxima. The error bars indicate standard deviations.

for both fixation conditions. In all conditions, there was significant $(P<0.001$, uncorrected) activation in Broca's region (Total: MNI coordinates $-52,14,22$, BA 44; $-62,20$,
-8 , pars orbitalis; total cluster size $k=1627$; BITE: $-60,4,26$, BA 44/6; $k=114$; FIX: $-44,12,18$, BA $44 ; k=96$; $-62,20,-8$, pars orbitalis; $k=19)$. The activation in Broca's region did 
not differ significantly (two-sample $t$-test, two-sided, $P<0.001$, uncorrected) between the two fixation conditions $[t(10)=4.04$ at $-66,16,10]$.

The mean time courses of each motion parameter for the total sample and FIX and BITE are displayed in Fig. 3. Figure 4 shows the mean(mean), mean(max), and max(max) values. The difference $\Delta$ between FIX and BITE was not significant for any parameter with respect to the mean (mean) head motion $(x: \Delta=0.10 \mathrm{~mm}, P=0.639 ; y: \Delta=0.30 \mathrm{~mm}$, $P=0.073 ; z: \Delta=0.13 \mathrm{~mm}, P=0.530$; pitch: $\Delta=0.006^{\circ}, P=0.432$; roll: $\Delta=0.001^{\circ}, P=0.876$; yaw: $\Delta=0.001^{\circ}, P=0.530$ ). No differences were observed for the mean $(\max )$ head motion either $(x: \Delta=-0.12 \mathrm{~mm}, P=0.639 ; y: \Delta=0.34 \mathrm{~mm}, P=0.149$; $z: \Delta=0.03 \mathrm{~mm}, P=0.755$; pitch: $\Delta=0.010^{\circ}, \quad P=0.530$; roll: $\Delta=0.003^{\circ}, P=0.755$; yaw: $\left.\Delta=-0.004^{\circ}, P=0.343\right)$. The mean overlap $(\bar{\Omega})$ of the volume of BA 44 before and after speaking was $\bar{\Omega}=98.1 \%$ for the total sample, $\bar{\Omega}=95.8 \%$ for the FIX group, and $\bar{\Omega}=100.0 \%$ for the BITE group. The Mann-Whitney $U$-test revealed a trend towards significance but no significant difference between the FIX and BITE groups $(P=0.063)$.

\section{Discussion}

We quantified the head movement caused by overt language production in fMRI. Normal fixation (FIX) was compared with the additional use of a bite-bar (BITE). The mean translation parameters were very small in both conditions and below the fMRI voxel size of $3 \times 3 \times 3 \mathrm{~mm}^{3}$. This implies that the signal of hardly any given voxel is erroneously assigned to one of its neighbours. The same holds for the maximum values that did not exceed $3 \mathrm{~mm}$ in any direction. Most interestingly, the use of a bite-bar did not reduce head motion any further. A trend towards significance was only observed for the translation in the $y$ direction and for the volume shift. The small absolute difference $\Delta$ between both groups, however, reveals the negligibility of this effect. This notion is further corroborated by the lack of a significant difference between the two head fixation methods in the functional imaging data in BA 44 in Broca's region.

The potential drawbacks of using a bite-bar are the additional disturbance of the participant caused by a dry mouth and sustained tension in the jaw muscles, which may interfere with the participant's attention to and performance of the actual task. Thus, considering the potential trade-off between minimally reduced head motion and this interference, the data speak in favour of simple head fixation with cushions and belts but without a bite-bar.

The average head motion parameters observed here correspond to data reported by Palmer et al. [7] and Gracco et al. [4]. Palmer et al. [7] compared overt with covert stem completion in fMRI. The mean head motion was smaller than $1 \mathrm{~mm}$ and did not differ between the two speech conditions. Gracco et al. [4] investigated overt sentence production in one fMRI study (study 1) with normal fixation and in another study (study 2) with an additional custombuilt head-restraining device. Whereas the motion parameters in study 1 were comparable to those of the FIX group in the present study, head motion in study 2 was more efficiently reduced with the head-restraining device without a bite-bar. The present study adds to these findings by statistically comparing two fixation methods in a larger sample, assessing the maximum head motion, and relating these findings to the cytoarchitectonically defined volume of BA 44 in Broca's speech region.

\section{Conclusion}

The data reveal the relatively small amount of head motion during overt language production. The effects advocate the use of standard head fixation or advanced head-restraint devices [4] but no bite-bar. Taken together, the results may encourage the use of overt speech in cognitive experiments, thus adding to the understanding of the function of the human brain.

\section{Acknowledgements}

The data were collected at the Max Planck Institute for Human Cognitive and Brain Sciences in Leipzig and analysed at the Research Centre Jülich.

\section{References}

1. Price CJ, Devlin JT, Moore CJ, Morton C, Laird AL. Meta-analyses of object naming: effect of baseline. Hum Brain Mapp 2005; 25:70-82.

2. Barch DM, Sabb FW, Carter CS, Braver TS, Noll DC, Cohen JD. Overt verbal responding during fMRI scanning: empirical investigations of problems and potential solutions. Neuroimage 1999; 10:642-657.

3. Birn RM, Bandettini PA, Cox RW, Shaker R. Event-related fMRI of tasks involving brief motion. Hum Brain Mapp 1999; 7:106-114.

4. Gracco VL, Tremblay P, Pike B. Imaging speech production using fMRI. Neuroimage 2005; 26:294-301.

5. Haller S, Radue EW, Erb M, Grodd W, Kircher T. Overt sentence production in fMRI. Neuropsychologia 2005; 43:807-814.

6. Heim S, Opitz B, Friederici AD. Broca's area in the human brain is involved in the selection of grammatical gender for language production: evidence from functional magnetic resonance imaging. Neurosci Lett 2002; 328:101-104.

7. Palmer ED, Rosen HJ, Ojemann JG, Buckner RL, Kelley WM, Petersen SE An event-related fMRI study of overt and covert word stem completion. Neuroimage 2001; 14:182-193.

8. Riecker A, Ackermann H, Wildgruber D, Dogil G, Grodd W. Opposite hemispheric lateralization effects during speaking and singing at motor cortex, insula and cerebellum. Neuroreport 2000; 11:1997-2000.

9. Amunts K, Schleicher A, Bürgel U, Mohlberg H, Uylings HBM, Zilles K Broca's regions revisited: cytoarchitecture and intersubject variability. J Comp Neurol 1999; 412:319-341.

10. Amunts K, Weiss PH, Mohlberg H, Pieperhoff P, Eickhoff S, Gurd JM, et al. Analysis of neural mechanisms underlying verbal fluency in cytoarchitectonically defined stereotaxic space: The roles of Brodmann areas 44 and 45 . Neuroimage 2004; 22:42-56. 